\title{
Mathematical model of the VAG gas valve identification algorithms
}

\author{
Adam Trojnar, MSc. Eng. \\ Institute of Applied Computer Science \\ Lodz University of Technology \\ ul. Stefanowskiego 18/22, 90-924 Lodz, Poland \\ Email: atrojnar@iis.p.lodz.pl
}

\author{
Piotr Ostalczyk, DSc. Eng. \\ Institute of Applied Computer Science \\ Lodz University of Technology \\ ul. Stefanowskiego 18/22, 90-924 Lodz, Poland \\ Email: piotr.ostalczyk@p.lodz.pl
}

\begin{abstract}
This paper present identification algorithms of the VAG gas valve and a comparison between them. One of the mathematical models is based on the differential-integral calculus of fractional order. This mathematical tool can be used for modeling devices serving to closed-loop systems synthesis. Admitting fractional orders in difference equations improves the performance of proportional-integral-derivative controller action.

Index Terms-gas valve, differential-integral cal-culus of fractional order, identification.
\end{abstract}

\section{INTRODUCTION}

T HE AUTOMATION determines a comfort of use and operating costs of the heating system, which is responsible for controlling its operation $[1,2,3]$. In discussed case the VC200 control unit is responsible for it, which along with the VAG gas valve constitutes a set to control the operation of heating boilers. This control unit is designed to maintain the degree of the valve opening based on measured temperature parameters and set temperature by the user. The valve offers linear and constant characteristics of operation of a gas burner to heating boiler with standby position. This solution causes a measurable reduction in gas consumption in older type of systems, elimination of incomplete burning and condensation of the exhaust fumes in emittors.

Many unpredictable factors must be taken into account in design of control systems. Application of coil and steel core in the VAG valve causes non-linear dependence of the degree of opening to current amplitude and hysteresis of valve motion up and down. In case of control with using information about the model, there is a possibility to remove uncontrolled disruptions. In PID type of control units the signal that controls the device is a sum of three functional blocks: multiplication, integration and differentiation. Difference, which appears between the set and currently measured value, is a result of specified operations. It is possible in more accurate way to describe real objects using differential equations of fractional order, and therefore application of fractional order control units in such cases is more beneficial $[1,2,3]$.

\section{IDENTIFICATION ALGORITHMS}

In a room was placed the test bench in order to study static and dynamic characteristics of the control system temperature. Setting parameters of the control unit operation is carried out by RS232 communication channel connected to the computer. The computer has installed the Windows operating system that is required to run the DIAG200 program. The test bench

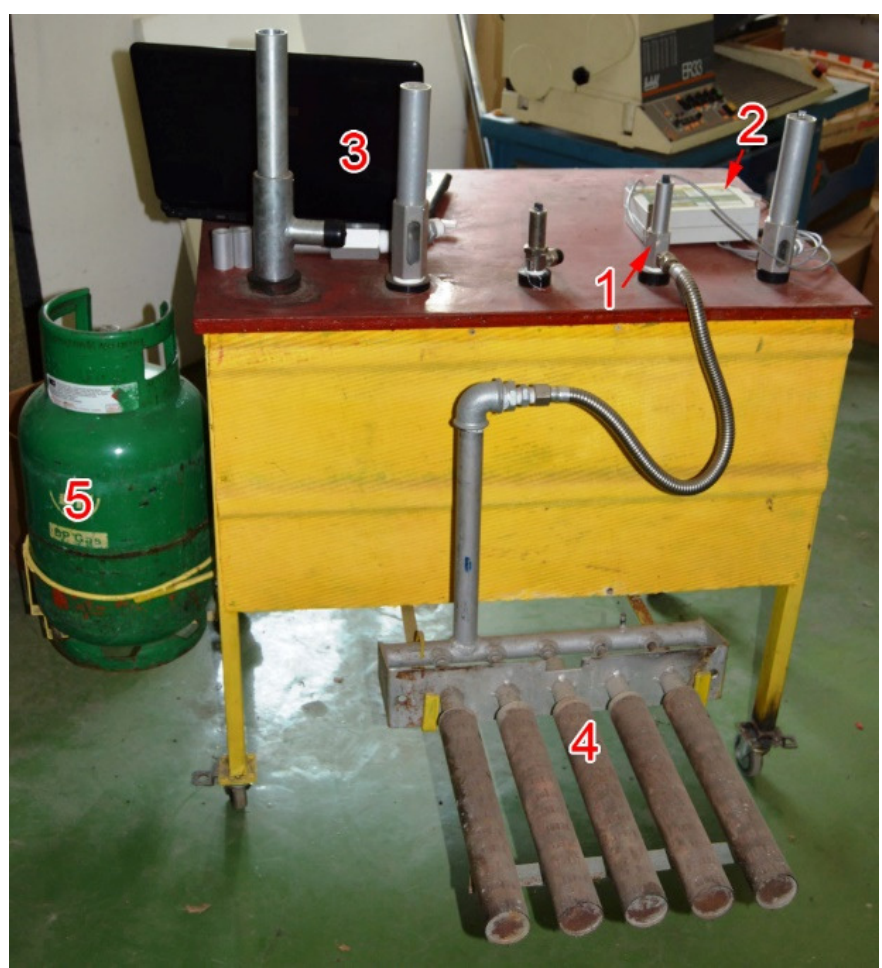

Fig. 1. The test bench for measuring the VAG gas valve.

consists of: 1. the VAG gas valve, 2.the VC200 controller, 3. a computer with the Windows OS, 4. a injector air nozzles, 5. a gas cylinder LPG.

Description of the black box which is a linear system having one input and output is described by a differential equations $[3,4]$. In this case, time is a independent variable and binding input values and the observed response. Measured system is described by the following differential equation:

$$
\frac{d x(t)}{d t}+a_{0} x(t)=b_{0} \frac{d u(t)}{d(t)}
$$


the solution of the Equation (1) is:

$$
x(t)=a e^{-b t}
$$

The difference backward $v$-th row is defined as:

$$
{ }_{k_{0}} \Delta_{k}^{(v)} x(k)=\sum_{i=k_{0}}^{k} a_{i-k_{0}}^{v} f\left(k+k_{0}-i\right)
$$

another form of the difference backward:

$$
k_{0} \Delta_{k}^{(v)} x(k)=\left[\begin{array}{llll}
a_{0}^{v} & a_{1}^{v} & \cdots & a_{k-k_{0}}^{v}
\end{array}\right]\left[\begin{array}{c}
x(k) \\
x(k-1) \\
\vdots \\
x(k)
\end{array}\right]
$$

To create the differential equation is applied the backward difference of first order $x(k)$ function:

$$
\begin{gathered}
{ }_{0} \Delta_{k}^{(1)} x(k)+a_{0} x(k)=b_{0} u(k) \\
x(k)-x[(k-1) h]+a_{0} x(k)=b_{0} \\
x(k)=\frac{1}{1+a_{0}}[x(k-1)]+\frac{1}{1+a_{0}}
\end{gathered}
$$

\section{A. Static model}

Static model can be described in a form of graph that presents static characteristics of studied object. The graph of this type is not dependent on the time. Static characteristics constitute a material for system identification; it is a relation between the input and output signal.

The controller uploads current temperature in the room from a sensor and according to parameters set by the program is controlled by a needle in the valve. This type of regulation enables to supply of planned quantity of gas to the injector air nozzles, and depending on demand to increase or reduce the temperature in examination room.

Study consisted on leading into the control system, unchanging in time, subsequent values of the input signal, and on measurement of corresponding values of the output signal. As a result of conducted experiment related to determination of the static characteristics, discreet values were obtained and they were marked on a graph (Fig. 2). Obtained graph of static characteristics shows the position of valve in relation to output voltage of the control unit.

The second basic aim in determination of static characteristics is to appoint characteristics equation from the graph (Fig. 2). Using computer synthesis methods and analyses there was a few methods to resolve the task. For the valve an approximation was used with method of the smallest squares. In order to obtain the static characterization of object, an approximation of received extortion values was conducted and corresponding responses on the graph (red line in Fig. 2). Below is presented third degree polynomial that approximates results of conducted measurement:

$$
w(x)=-x^{3}+0,0005 x^{2}+0,0322 x+15,579
$$

In order to describe appearing phenomena and processes there are used mathematical models. Complexity result of identification depends on its destination. It is possible to describe

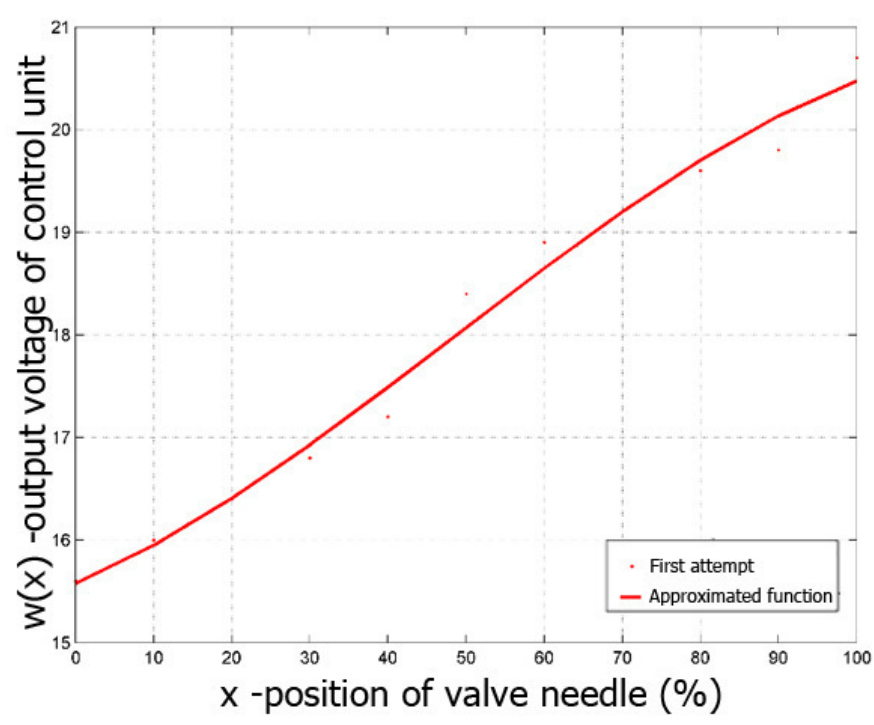

Fig. 2. Graph of static characteristics shows the position of valve in relation to output voltage of the control unit.

its correct construction by mapped accuracy of the process flow with reference to applied model. On the one hand, too high complexity of the model structure may require a lot of calculation by which the model may turn out to be useless. However, on the other hand, the simplified model may have large variations in relation to mapped reality [5].

\section{B. Dynamic models}

An important aspect is identification of dynamic properties of the object. Observation of the process response to stimulation enables to obtain information about its description in the field of variable time. Time line of the output rate triggered by extortion is called dynamic characterization of the object. Dynamic characterization is a transmittance between two established states. Method of dynamic model identification in case of valve consists in evaluation of the object transmittance based on variable characterization. The object of regulation is a movable control needle fixed on the electromagnet core in the VAG valve. As the variable characterization of valve a percentage relation of needle fall was accepted, when set temperature and measured temperature starts to reach standby position.

Temperature in the room, which is the object of regulation, at the time of measurement was $17^{\circ} \mathrm{C}$. On the controller was set temperature $23.5^{\circ} \mathrm{C}$. After reaching required temperature the valve began to limit supply of gas to the burner by linear needle fall. Figure shows the moment of needle fall to full opening to a minimal position. Duration of a single experiment was $33 \mathrm{~s}$, results of sampling every $1 \mathrm{~s}$. were presented in points. Figure presents three selected sample measurements, differences between individual measurements result from carrying out examining within the entire day. As the variable characterization of valve a percentage relation of needle fall was accepted. 


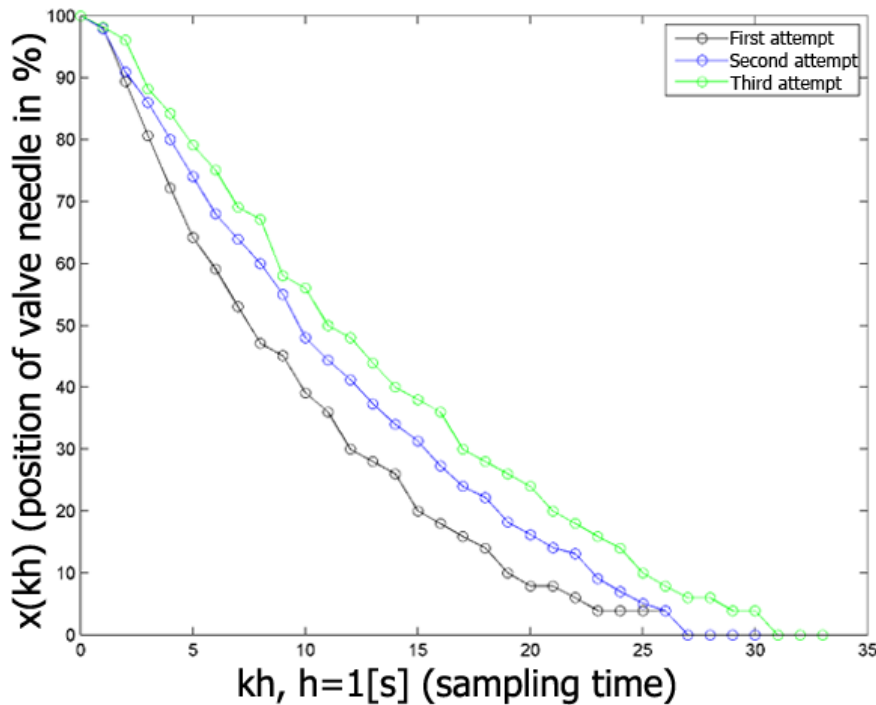

Fig. 3. Measurement of the valve responses to reduce the temperature.

Based on the Fig. 3 it is possible to state that it is the inert object of first order. Inert response type of regulation object to irregular extortion results from the process occurring during the study. Extortion in reductions of opening degree of the valve causes a change of gas supply flow. Reduced power of injector air nozzles resulting for this reason proceeds with certain delay. Other processes are also delayed: heat transfer between the burner and examination room via air and heat transport from surrounding to the temperature sensor. After leveling of new heat loss value in the room with the amount of heat provided by the burner arise a new steady-state and air temperature, and a level of the needle position remains unchanged.

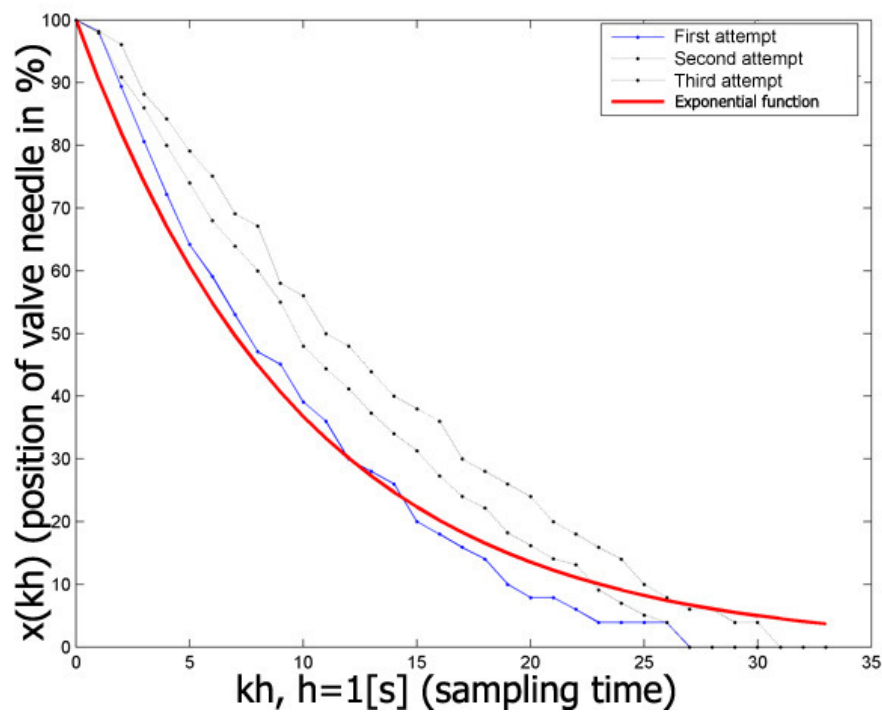

Fig. 4. Exponential function approximate results of the first measurement.

In the measured discreet system described by Equation (2) where continuous time $t$ must be replaced by the value $k$ which is next step, the $h$ value is a sampling time and equal 1 second. On obtained values was approximated exponential function:

$$
x(k h)=a e^{-b k h}
$$

To the subsequent measurements was matched functions. For the first attempt of response was approximated and illustrated in Fig. 4 exponential function: $x(k)=a e^{\frac{-k}{10}}$, the second attempt of response: $x(k)=a e^{\frac{-k}{12}}$, the third attempt of response: $x(k)=a e^{\frac{-k}{14}}$.

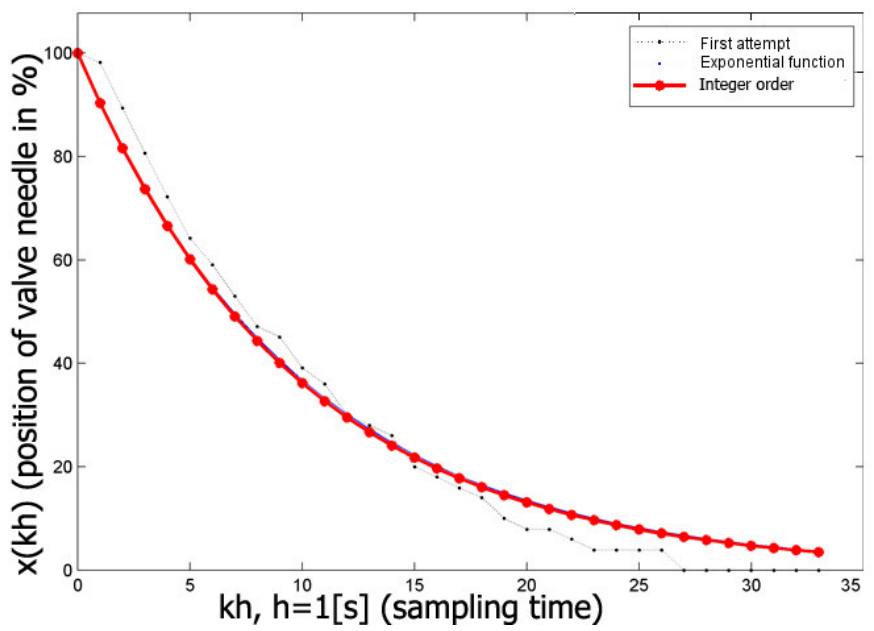

Fig. 5. Function is based on differential equation of the integer order which aproximate the results of the first test measurements.

The second dynamic model is described by a discrete transfer function and the state-space equations [3]. Equation (1) is approximated by the backward difference of first order:

$$
\begin{aligned}
& \frac{\Delta x(k h)}{h}+a_{0} x(k h)=b_{0} \frac{\Delta u(k h)}{h} \\
& \Delta x(k h)+a_{0} h x(k h)=b_{0} \Delta u(k h)
\end{aligned}
$$

the difference of function $f(k h)$ is:

$$
\Delta f(k h)=f(k h)-f[(k-1) h]
$$

the difference of function (12) is substituted to the backward difference of first order (11). Based on fact that the measured object got the Dirac delta function extortion it follows $\Delta u(k h)$ is replaced by $\delta(k h)$ :

$$
\begin{gathered}
x(k h)-x[(k-1) h] a_{0} h x(k h)=b_{0} \delta(k h) \\
\left(1+a_{0}\right) x(k h)=x[(k-1) h]+b_{0} \delta(k h)
\end{gathered}
$$

The solution of the approximation which is based on the backward difference of first order is:

$$
x(k h)=\frac{1}{1+a_{0}} x[(k-1) h]+\frac{b_{0}}{1+a_{0}} \delta(k h)
$$

The next step is to adjust the parameters $a_{0}, b_{0}$ in order to reproduce the response. The approximate function is based 
on the differential equation of integer order (Fig. 6) where: order of differential equation for all attempts $v=1$ and rate $b_{0}$ of differential equation is equal $v$. Rates of differential equation for the first attempt: $a_{0}=0,107$; for the second attempt $a_{0}=0,085$; for the third attempt $a_{0}=0,073$.

The third dynamic model is also described by a discrete transfer function and the state-space equations. The differ-integral of fractional order is a generalization of the calculus[3]. Equation (1) is approximated by the backward difference of fractional order:

$$
\frac{\Delta^{v} x(k h)}{h}+a_{0} x(k h)=b_{0} \frac{\Delta u(k h)}{h}
$$

The solution of the approximation which is based on the backward difference of fractional order is:

$$
\left[\begin{array}{llll}
1 & a_{1}^{v} & \cdots & a_{k}^{v}
\end{array}\right]\left[\begin{array}{c}
x(k h) \\
x(k-1) h \\
\vdots \\
x(h)
\end{array}\right]+a_{0} x(k h)=b_{0} \delta(k h)
$$

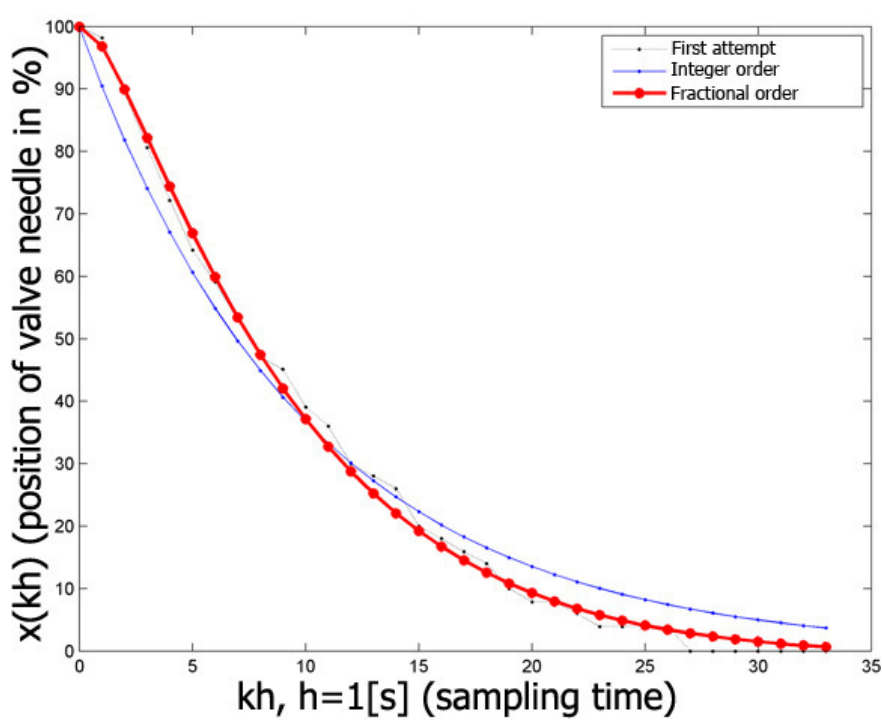

Fig. 6. Function based on the differential equation of fractional order.

The next step is to adjust the parameters $a_{0}, b_{0}, v$ in order to reproduce the response. Function based on differential equation of fractional order (Fig. 6), where: rate $b_{0}$ of differential equation for all attempts is equal $v$. Order of differential equation for the first attempt $v=1,08$, rates of differential equation: $a_{0}=0,115$; Order of differential equation for the second attempt $v=1,08$, rates of differential equation: $a_{0}=0,092$; Order of differential equation for the third attempt $v=1,08$, rates of differential equation: $a_{0}=0,073$.

In order to exclude the role of subjective factors in assessing the accuracy of model, as a basis it is necessary to adopt measurable criterion. It is possible to evaluate the level value of control system using integral rates of the course of regulated size. In case of conducted study of valve response, the model accuracy consists on comparison to what extend modeled functions correspond to real measurements. As the correctness criterion of model selection a value of the integral was chosen from the Integral Square Error between measured values and mathematical model [5].

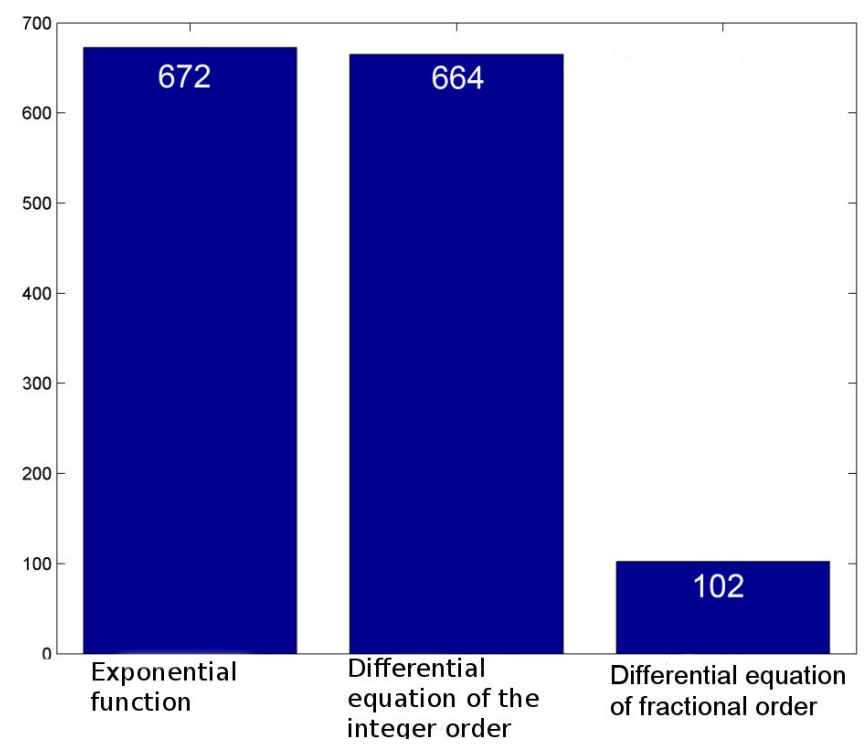

Fig. 7. Comparision a quality of the identification models.

Based on above graphs it is possible to conclude that in any case the best response of device reflects a model based on differential equation of fractional order. The model that reaches the best results in each comparison is several times better than other. Models based on exponential function and differential equation of first order reaches similar degree of approximation.

\section{REFERENCES}

[1] YaLi He, RuiKun Gong, Application of Fractional-order Model Reference Adaptive Control on Industry Boiler Burning System. Intelligent Computation Technology and Automation (ICICTA), 2010.

[2] Piotr Duch, Optymalizacja algorytmów numerycznych wykorzystujacych równania różnicowe całkowitych i niecałkowitych rzédów, Praca doktorska, Politechnika Łódzka, 2014, pp 589 - 594 (in Polish).

[3] Piotr Ostalczyk, Zarys rachunku rózniczkowo-całkowego ułamkowych rzedów. Teoria i zastosowania w automatyce, Wydawnictwo Politechniki Łódzkiej, 2008, pp 1 - 25, 161 - 163, 199 - 209 (in Polish).

[4] Piotr Ostalczyk, Variable-, fractional-order discrete PID controllers, Methods and Models in Automation and Robotics (MMAR), 17th International Conference, 2012, pp 534 - 539.

[5] Piotr Ostalczyk, Piotr Duch Closed - Loop system synthesis with the variable-, fractional - Order PID controller, Methods and Models in Automation and Robotics (MMAR), 17th International Conference, 2012, pp 589 - 594.

[6] Richard Banach, Pieter Van Schaik, Eric Verhulst Simulation and Formal Modelling of Yaw Control in a Drive-by-Wire Application, Computer Science and Information Systems (FedCSIS) 2015, pp 731 - 742. 